\title{
EDUCACIÓN Y DESCENTRALIZACIÓN. VIRTUDES, DEBILIDADES Y PROPUESTAS EN EL CASO CHILENO.
}

\section{Leonardo Letelier S.}

\author{
Universidad de Chile
}

\section{Resumen}

Si bien la descentralización parece ofrecer un camino de fortalecimiento de la calidad de la educación pública, la literatura sobre el particular no es específica en señalar la forma concreta que ésta debe tomar. No obstante lo anterior, este trabajo plantea que existen tres elementos o requisitos básicos que deben ser resguardados en el diseño de un sistema de gestión integral descentralizada de los colegios públicos. Estos son la rendición de cuentas de las autoridades a cargo, el control sobre los costos por parte de las mismas autoridades y la capacidad de la autoridad competente para administrar su estructura de gastos. Atendiendo estas condiciones, se propone la implementación de distritos escolares financiados mediante transferencias no condicionadas desde el nivel central de gobierno.

Palabras Claves: Educación, Descentralización, Economía Política, Chile

\section{Abstract}

While decentralization seems to provide a way to strengthen the quality of public education, the existing literature on the subject matter is far from being specific in pointing out the concrete way in which this has to be done. Regardless of the aforementioned hypothesis, this present paper states that three basic requirements are to be ensured in the process of decentralizing this function of the State. They are the accountability of those in charge, the control over costs by these same authorities and their real capacity to decide on the structure of expenditures to be made. In this context, this paper proposes the implementation of school districts in Chile, which are to be funded by unconditional grants from the central government.

Keywords: Education, Decentralization, Political Economics, Chile 


\section{Introducción}

Debemos entender como descentralización la concesión de un mayor grado de libertad a los niveles subnacionales de gobierno para ejecutar su gasto en función de las preferencias políticas del votante mediano local y/o de generar recursos propios a través de la implementación autónoma de tasas y bases tributarias. Sin embargo, es igualmente legítimo preguntarse qué debemos incluir en el espacio de autonomía exclusivo de cada jurisdicción. Muchas y muy diversas son las dimensiones de este mismo gasto, incluyendo elementos tan distintos como las decisiones de política económica en torno a temas que son propios del nivel local (estadual, provincial o regional), y/o cuestiones normativas específicas como puede ser la elaboración de un plan regulador o la elaboración de la malla curricular de la propia educación primaria o secundaria administrada por el municipio.

Existe abundante evidencia empírica en favor de la hipótesis según la cual la descentralización -genéricamente concebida- mejora la calidad del gasto público en educación (Letelier, 2004). № obstante lo anterior, esta misma evidencia es poco conclusiva y usualmente vaga en lo referente a la forma concreta que dicha descentralización debe adoptar. Este trabajo pretende examinar las bondades y debilidades del proceso de descentralización de la educación en Chile, el cual fue iniciado a comienzo de los años 80 . Se plantea que muchos de los problemas de gestión vinculados a la educación pública chilena se originan en que la descentralización referida ha sido mal diseñada, y que un cambio deseable sería la creación de "distritos escolares" que sustituyan la actual dependencia de los establecimientos respecto de los municipios. En la práctica, esto supone profundizar la descentralización, acercando más la gestión local respecto de los ciudadanos.

Un aspecto igualmente central es la cuestión de cómo asignar competencias entre niveles de gobierno. Éste ha sido un problema poco explorado en la teoría económica moderna, habiendo pocas referencias normativas concretas en torno al tema. La más conocida es aquella sugerida por Musgrave (1959) en su clásico tratado de finanzas públicas, y revitalizada por el conocido texto de Oates (1972). El principio general por ellos consagrado establece que la función de asignación de recursos es aquella en la cual los niveles descentralizados de gobierno poseen ventajas objetivas de gestión respecto del nivel central. Las funciones de estabilización económica y redistribución del ingreso inducen efectos externos (externalidades) a la propia jurisdicción en la cual son ejecutadas, provocando un resultado global subóptimo cuando tales decisiones son tomadas descentralizadamente.

El resto de este artículo presenta la experiencia internacional en la segunda sección y una discusión sobre el caso chileno en la tercera sección. La propuesta de diseño competencial se desarrolla en la cuarta sección. 


\section{La experiencia internacional comparada}

Como suele suceder con todos los ámbitos de las políticas públicas, la experiencia internacional es diversa, difícil de comparar y si bien de ella pueden extraerse interesantes lecciones generales, poco se puede concluir en cuanto a los aspectos específicos de cada caso. Si hubiese que identificar una tendencia, ésta ha sido sin duda favorable a la descentralización en el ámbito educacional. En América Latina, los casos de Bolivia y Colombia son muy citados y estudiados. Fauget y Sanchez (2008) muestran que en el caso boliviano, la descentralización generó un cambio significativo en el patrón de inversión pública a favor de la educación. $E$ mismo estudio sugiere que los municipios colombianos "acreditados", los cuales poseen más libertad para ejecutar recursos públicos, han sido los más exitosos en mejorar la asistencia a clases de los estudiantes en los colegios públicos. La experiencia argentina parece indicar que la descentralización de la educación secundaria desde el nivel federal hacia las provincias, la cual tuvo lugar entre 1992 y 1994, indujo un mejoramiento general en los resultados de las pruebas de matemáticas e idiomas para los llamados "colegios descentralizados", aun cuando tales beneficios fueron solo significativos para las comunidades más ricas (Galiani, et al., 2008). En el caso chileno, Chang y Urquiola (2006) muestran que si bien el modelo chileno de financiamiento aumentó considerablemente la capacidad de los padres para elegir el colegio que mejor se ajustaba a sus preferencias - produciendo una masiva migración de estudiantes desde establecimientos públicos a los privados-, no existe evidencia clara en torno al impacto sobre la calidad de la educación. Sin embargo, los mismos autores advierten sobre posibilidad de que la libre elección de los padres haya resultado en mejoras en el bienestar difíciles de cuantificar.

Interesante resulta constatar que estudios similares suelen ser menos optimistas en cuanto a la descentralización se refiere en países de mayor nivel de desarrollo. En un análisis sobre el caso suizo, Barankay y Lockhood (2007) concluyen que los municipios con mayor autonomía financiera tienen mejor desempeño. Sin embargo, el mismo trabajo sugiere que los resultados son sensibles a la capacidad técnica de los funcionarios municipales. Menos claro es el caso de Suecia, país en el cual tuvo lugar un importante proceso descentralizador de la educación pública secundaria entre los años 1991 y 1996. Ahlin y Mork (2008) arrojan evidencia a favor de la hipótesis de que tanto los recursos destinados por los municipios suecos luego de la descentralización, como así también otros indicadores de calidad de los establecimientos, no experimentaron cambios importantes luego de concluido el período de reformas.

Este breve recuento en torno a la evidencia disponible solo permite extraer lecciones generales y deja por tanto varias preguntas centrales sin responder. La lección número 1 es que si bien la evidencia observada parece indicar que la descentralización afecta favorablemente la 
calidad de este importante servicio público, la interpretación del resultado depende críticamente de la (o las) variable(s) sobre la(s) cual(es) desarrollamos el análisis empírico. En este aspecto, la dificultad de comparar los trabajos aquí consignados solo permite decir que (lección número 2): la descentralización no parece ser neutral respecto de sus consecuencias en el desempeño de los colegios. En cuanto a las preguntas abiertas y aún no respondidas, ellas giran en torno al significado de la descentralización en el tema que nos ocupa. En el entendido de que el fenómeno en cuestión admite múltiples dimensiones, cabe concluir que la evidencia disponible dista de ser concluyente respecto a qué dimensión específica es aquella sobre la cual debemos descentralizar. ¿Qué nivel de gobierno -supuestamente subnacional- debe asumir la función de administrar los establecimientos? ¿Debe ser este un distrito cuyas competencias se focalizan en una función única, o debe ser un municipio con un amplio rango de funciones generales? Junto con esto, debemos diseñar un sistema que defina en forma transparente, eficiente y clara cómo se asignarán los recursos provenientes del gobierno central para financiar la educación. ¿Lo haremos con un voucher por alumno -como en el caso chileno (ver sección siguiente)- o como sucede en la mayor parte de los países occidentales desarrollados en que hay una combinación de factores?

\section{Aspectos específicos del caso chileno}

\subsection{El marco general}

Chile ha sido un país pionero al introducir un sistema de financiamiento de la educación escolar pública a través de un voucher por alumno, hecho que fue acompañado por el traspaso de los establecimientos escolares a la administración municipal al inicio de los años 80 . El apoyo estatal a la educación opera con un financiamiento semejante, paralelo al sistema municipal, en la forma de los llamados colegios "privados subvencionados", los cuales reciben también un voucher por alumno, pero en proporción inversa al cobro de matrícula de tales establecimientos. Estos están llamados a constituirse en un factor adicional de incentivo sobre el desempeño de la educación municipal, toda vez que compiten por alumnos financiados por el Estado.

La lógica del sistema chileno se basa en el viejo modelo del "voto con el pie" (Hrichman, 1970), el cual predice que si los beneficiarios del sistema pueden elegir libremente dónde educarse, migrarán hacia las jurisdicciones (y establecimientos) que ofrecen las mejores condiciones. Este mecanismo penalizará a los gobiernos locales (estaduales, provinciales), cuya capacidad de gestión es más débil, y premiará aquellos más exitosos por la vía de incrementar su matrícula. 


\subsection{Los problemas del sistema chileno}

Se plantean a continuación tres problemas básicos del modelo chileno de educación municipal. El primero es la relación contractual con los profesores, la cual genera un flujo de gastos e ingresos que es inconsistente con el espíritu original del modelo, basado en el financiamiento en base a un voucher por alumno. El segundo es la coherencia político-institucional del sistema. Ello supone reflexionar sobre la economía política del problema y sus implicancias sobre los resultados. El tercer problema se refiere a la equidad horizontal entre municipios.

\subsubsection{La relación contractual con los profesores}

La forma específica en la cual se celebran los contratos de trabajo con el estamento docente representa un elemento central del problema que nos ocupa. Una genuina descentralización de la educación supone un traspaso de esta responsabilidad al nivel de gobierno responsable de administrar la educación. Éste es, por ejemplo, un rasgo común de casos tan diversos como los de Argentina y Suecia, en los cuales se experimentó un drástico proceso descentralizador en el curso de los años 90. Mientras que en Argentina esta responsabilidad fue delegada a las provincias, los municipios suecos negocian directamente con los profesores, desde 1996.

En la experiencia chilena, los contratos laborales de los profesores fueron realizados en base al Código del Trabajo, hasta 1991. Dicho marco legal es utilizado en el contexto de las relaciones laborales entre privados. A partir de ese año, la institucionalidad vigente para los profesores municipales pasó a regirse por el llamado Estatuto Docente, el cual restringe severamente el margen de maniobra de los propios municipios para negociar las condiciones de trabajo de los profesores. Lo anterior es reconocido como un problema de gran trascendencia en la administración municipal de la educación, toda vez que enfrenta a los municipios con costos fijos por concepto de remuneraciones en razón del estatuto referido, los cuales deben ser financiados con ingresos variables a través del voucher por alumno.

El problema en cuestión posee severas implicancias sobre la coherencia de la descentralización, como así también sobre la apropiada estructura de incentivos que debe regir en beneficio de una educación de calidad. En principio, no existen razones de fondo para argumentar que los profesores deban tener un régimen laboral independiente de otros profesionales y/o funcionarios. Sin embargo, parece evidente que la transferencia de responsabilidad en materia de educación debe acompañarse con una dosis equivalente de flexibilidad en la administración de sus recursos, entre los cuales el "recurso humano" es el más importante.

\subsubsection{La Economía Política del problema}

Una cuestión de fondo en el tema referido es la pertinencia del municipio como el ámbito competencial apropiado para la educación. No obstante la evidencia empírica antes mencionada, 
el argumento teórico a favor de la descentralización que más nítidamente refleja este punto es aquel sobre la rendición de cuentas de los responsables de la función en cuestión. ¿Será, en verdad, el esfuerzo del propio gobierno municipal en favor de una buena gestión un factor determinante en el éxito político de la administración municipal? Tal sería el caso si la buena gestión en este ámbito específico de la educación fuera determinante de la reelección de la coalición y/o el partido en ejercicio. Expresado en términos del conocido trabajo de Seabright (1996), lo que está en juego es el hecho de si el éxito en educación aumenta la probabilidad del incumbente de ser reelegido.

En ausencia de externalidades entre municipios, la referida rendición de cuentas se enmarca en el juego político de las autoridades locales. La forma más nítida de verlo es apelando al hecho de que, al ejercer su voto, los electores del nivel nacional de gobierno tendrán dificultades evidentes para identificar la responsabilidad del gobierno en ejercicio en lo referente a la gestión de la educación a nivel de cada jurisdicción. La decisión de quien vota, estará inevitablemente contaminada por una amplia gama de consideraciones, entre las cuales la educación es solo una más. A contrario sensu, la gestión local de la educación permite al elector local -el cual debe elegir autoridades para su propia jurisdicción- penalizar con mayor propiedad los méritos (y deméritos) del nivel local, en lo que a educación se refiere. En suma, la administración descentralizada de la educación genera mayor rendición de cuentas sobre los responsables de su administración, e induce por lo tanto, un especial incentivo en beneficio de una buena gestión.

Una dimensión poco explotada del problema, si bien muy evidente a la luz de la importancia vital de la educación en el contexto de las políticas públicas, es el hecho de que los municipios chilenos co-financian la educación. Abundante es la literatura que pone en tela de juicio cualquier proceso de descentralización que apunte a descentralizar la llamada "función redistributiva" del Estado (Musgrave, 1959, Oates, 1972). Si los beneficiarios tienen capacidad de "votar con el pie", ello redundará en una inconsistencia fundamental en la estructura de incentivos en el esfuerzo por promover políticas de orientación social. La descentralización producirá un resultado paradójico, toda vez que las jurisdicciones cuyo esfuerzo por canalizar recursos en favor de objetivos tales como educación, salud, o cualquier otra área similar, serán objeto de un mayor grado de migración desde localidades cercanas, agudizando aún más el problema original. En la experiencia chilena, ello se expresa en el hecho de que muchos municipios ricos reciben un significativo contingente de alumnos que provienen de comunas vecinas, en las cuales, por problemas de disponibilidad de vacantes y/o calidad de los establecimientos, las familias residentes prefieren enviar a sus hijos a colegios municipales de comunas con un mejor servicio educacional. Es evidente que ello reduce severamente la rendición de cuentas mencionada, y con ello también el esfuerzo del sistema municipal en su conjunto por fortalecer la función en cuestión. Al momento de votar, muchos de los beneficiarios no serán residentes locales. 


\subsubsection{El problema del financiamiento}

En el contexto del tema que nos ocupa, cabe hacer notar un rasgo muy particular de América Latina, del cual Chile no está ausente. Se trata de la desigualdad territorial de los recursos fiscales. Por razones históricas difíciles de identificar, la región ha sido tradicionalmente muy centralizada vis a vis los casos de Europa y Estados Unidos. Ello constituye un ingrediente que condiciona severamente los resultados de la descentralización, puesto que exige la implementación de sistemas muy agresivos de igualación fiscal a través de transferencias intergubernamentales, a fin de garantizar un estándar mínimo en la prestación de servicios. Lo anterior revela una importante debilidad del sistema chileno de financiamiento de la educación pública municipal. Esta se basa en un voucher por alumno, más un importante número de programas de apoyo con fines específicos diseñados por el Ministerio de Educación. Nos concentraremos en el voucher por alumno como la base del sistema. En este contexto cabe preguntarse el impacto que la evidente heterogeneidad entre municipios tiene sobre el gasto efectivo por estudiante. La Figura 1 permite apreciar la magnitud de esta heterogeneidad en la capacidad de los municipios para hacer aportes al financiamiento de la educación. El eje de las ordenadas presenta el porcentaje del total de recursos disponibles para educación aportados por el propio municipio. Se constata claramente que los municipios con mayor aporte son en general los que poseen mayores recursos por habitante. Este desequilibrio adquiere una connotación aun más dramática si se considera que son precisamente los municipios más pobres aquellos en los cuales el aporte del propio municipio es menor.

FIGURA 1: Aporte Municipal al Gasto Total en Educación y Presupuesto Municipal por Habitante

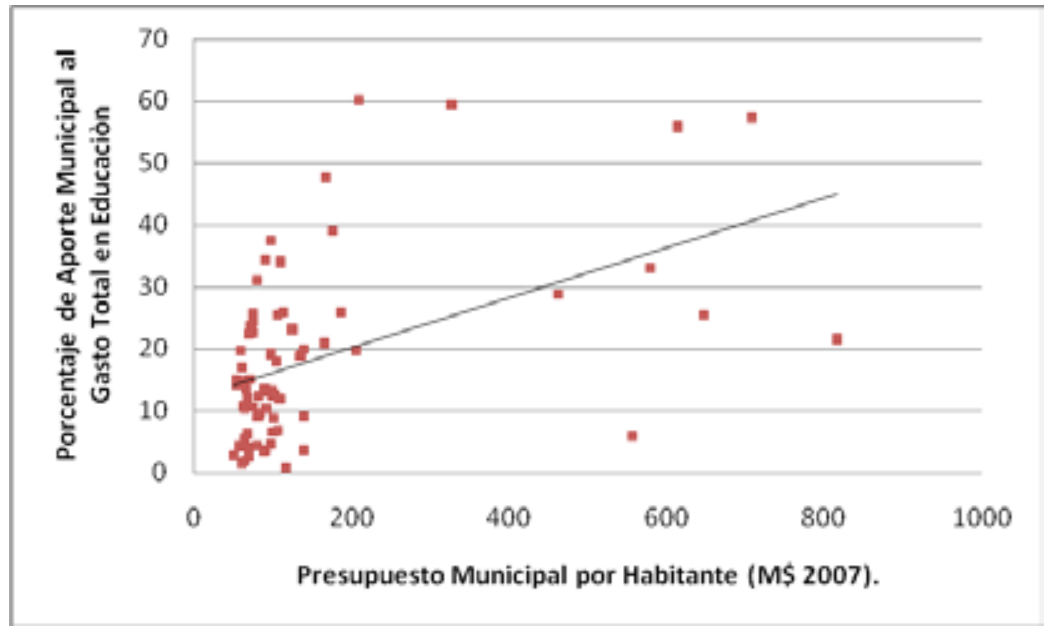

Fuente: Elaboración propia en base a datos de la Subsecretaría de Desarrollo Regional 
El corolario de este conocido problema es muy directo y claro. Si bien la descentralización de la educación es una política genéricamente favorable en el desempeño de la educación pública, deben distinguirse la descentralización de la gestión, y por lo tanto del gasto, de aquella respecto del financiamiento. En el caso chileno, una parte de tal financiamiento proviene en efecto de los propios municipios. Es precisamente este factor el que induce la heterogeneidad que exhibe la Figura 1, y que a la vez se ve contaminada con la existencia de externalidades entre jurisdicciones vecinas. Dado que tales externalidades son positivas, debemos esperar que el diseño de financiamiento vigente en Chile genere un nivel subóptimo de gasto en educación a nivel agregado, toda vez que el beneficio marginal de un peso más en este rubro no es completamente internalizado por la unidad territorial que lo gasta.

Un elemento complementario al problema anterior es el hecho de que si los municipios contribuyen con su presupuesto al financiamiento de la educación, existe poca claridad en torno al efecto real de incrementar el valor del voucher por parte del nivel central de gobierno. Bajo condiciones de restricción al crédito, tal como es el caso municipal chileno, un mayor financiamiento del gobierno central a la educación municipal, podría generar un desincentivo de los propios municipios en beneficio del aporte que estos realizan a la educación. Vale decir, el aporte marginal de un peso más del gobierno central tendrá un efecto igual o menor a uno en el gasto real en educación a nivel municipal. Existe, por lo tanto, un potencial "crowding-out" de recursos municipales por efectos del mayor aporte del nivel central. Si bien no hay mediciones de este efecto, parece razonable hipotetizar que tal es el caso.

\section{Una propuesta de mejoramiento}

El problema central de asignación de competencias entre niveles de gobierno y del modelo de financiamiento debe resguardar tres elementos básicos. El primero es que el grado de rendición de cuentas respecto de la función de gobierno en cuestión, en este caso la educación pública primaria y secundaria, sea la máxima posible. Ello supone garantizar que los electores podrán penalizar con su voto la eventual mala gestión, y premiar si este es el caso, a las autoridades exitosas en esta misma gestión. Inevitablemente, lo anterior exige transparencia en la administración de los colegios, y autoridades democráticamente elegidas por la comunidad. La descentralización de la educación sirve precisamente a este propósito, puesto que la gestión del gobierno municipal es fácilmente visible y susceptible de evaluar por quienes eligen autoridades locales. La cuestión de fondo es si tal grado de rendición de cuentas puede ser en verdad fortalecido. La respuesta es que sí, en la medida que la educación pueda ser administrada por gobiernos locales unifuncionales. Estos se denominan "distritos", y ocupan un importante lugar en la administración pública de países desarrollados. Un distrito 
educacional con autoridades elegidas, podría constituir un paso interesante en la dirección de mejorar la calidad de la educación. Debe entenderse que esta propuesta supone un grado mayor -y no menor- de descentralización. Resulta pertinente preguntarse por qué la educación debiese administrarse en el marco de un distrito y en cambio otros servicios básicos, tales como el alumbrado público, la recolección de basura, la policía y otros pudiesen estar exentos de esta figura especial. La respuesta pasa por una opción política, si bien la justificación teórica puede encontrarse en el concepto de los llamados "bienes meritorios", los cuales constituyen servicios de alta valoración social cuya naturaleza amerita excepciones respecto de los demás.

El segundo elemento central a considerar es que en el caso chileno, inevitablemente el gobierno central, y no el gobierno local, debiese proveer el grueso del financiamiento a la educación pública básica y media. Lo anterior exige diseñar un sistema de transferencias que identifique el nivel de gasto local en educación necesario para proveer un nivel de servicio que se ajuste a un estándar mínimo garantizado a través del territorio. Idealmente, y precisamente a fin de fortalecer el grado de rendición de cuentas antes referido, las transferencias al distrito por este concepto deben ser no condicionadas a usos específicos del rubro educacional, de suerte que las autoridades locales puedan gestionar con libertad los recursos recibidos. A fin de evitar los efectos externos (externalidades) a la jurisdicción, ésta debe atender solo a educandos provenientes del distrito.

El tercer elemento a considerar en el diseño es que las autoridades a cargo deben tener control sobre los costos de administrar los colegios. Es evidente que la legislación laboral vigente en Chile, basada en el Estatuto Docente, dista de permitir este grado de autonomía. Solo la debida flexibilidad para sancionar profesores de mal desempeño, reasignar docentes entre establecimientos y cambiar el tamaño y la estructura del personal, permiten aprovechar a plenitud una genuina descentralización.

\section{Conclusiones}

El presente ensayo ofrece una discusión en torno a los efectos de la descentralización en el desempeño de la educación pública. Se argumenta que si bien la descentralización, genéricamente definida, parece ofrecer un camino de fortalecimiento en la calidad de este importante servicio público, la literatura sobre el particular no es específica en señalar la forma concreta que esta debe tomar. No obstante lo anterior, se señala que existen tres elementos 0 requisitos básicos que deben ser resguardados en el diseño de un sistema de gestión integral de los colegios públicos. Estos son la rendición de cuentas de las autoridades a cargo, el control sobre los costos por parte de las mismas autoridades y la capacidad de la autoridad 
competente para administrar su estructura de gastos. Esto último exige modificar el actual Estatuto Docente.

En el contexto de un amplio espectro de posibilidades en cuanto al diseño del ente territorial y/o la instancia administrativa responsable, el presente trabajo propone la implementación de un distrito escolar como el nivel de gobierno encargado de la función en cuestión. Este debe tener autoridades elegidas por la comunidad y una sola función: la de administrar los colegios que hoy se encuentran bajo el alero municipal. En materia de financiamiento, se propone un sistema de transferencias no condicionadas hacia los distritos. 


\section{Referencias Bibliográficas}

Ahlin A. y E. Mork. 2008. Effects of Decentralization on School Resources. Economics of Education Review 27 (3): 276-284.

Barankay I. y B. Lockwood. 2007. Decentralization and the Productive Efficiency of Government: Evidence from the Swiss Cantons. Journal of Public Economics 91 (5-6): 1197-1218.

Faguet J.P. y F. Sánchez. 2008. Decentralization's Effects on Educational Outcomes in Bolivia and Colombia. World Development 36 (7): 1294-1316.

Galiani S., P. Gertler y E. Schargrodsky. 2008. School Decentralization: Helping the Good get Better, but Leaving the Poor Behind. Journal of Public Economics 92 (10-11): 2106-2120.

Chang-Tai H. y M. Urquiola. 2006. The Effects of Generalized School Choice on Achievement and Stratification: Evidence from Chile's Voucher Program. Journal of Public Economics 90 (8-9): 1477-1503.

Hirschman, A. O. 1970. Exit, Voice, and Royalty. Cambridge: Harvard University Press.

Letelier, S. L. 2004. Fiscal Decentralization as a Mechanism to Modernize the State. Journal of Institutional Comparisons 2 (1): 15-20.

Musgrave R. 1959. The Theory of Public Finance. New York: McGraw-Hill.

Oates, W. 1972. Fiscal Federalism. New York: Harcourt, Brace Jovanovich.

Seabright, P. 1996. Accountability and Decentralisation in Government: An Incomplete Contracts Model. European Economic Review 40: 61-89.

Leonardo Letelier S. es Director (S) de las Escuelas de Pregrado y Postgrado del Instituto de Asuntos Públicos de la Universidad de Chile. Es PhD in Economics por la University of Sussex, MPill in Economics por la University of Cambridge, Magíster en Economía e Ingeniero Comercial, por la Universidad de Chile. Su principal área de investigación es la Descentralización Fiscal. Entre sus trabajos en ese campo, destaca "Explaining Fiscal Decentralization", publicado en Public Finance Review 33 (2), 2005.

E-Mail: lletelie@uchile.cl 
